\title{
Probing Supercritical Accretion in Ultraluminous X-ray Source M82 X-1 by means of X-ray Spectral Evolution Ana- lysis
}

\author{
Izatul Hafizah ${ }^{1, *}$ and Kiki Vierdayanti ${ }^{2, * *}$ \\ ${ }^{1}$ Astronomy Study Program, Faculty of Mathematics and Natural Sciences, Institut Teknologi Bandung, \\ Ganesha 10, Bandung, 40132, Indonesia \\ ${ }^{2}$ Astronomy Research Division, Faculty of Mathematics and Natural Sciences, Institut Teknologi Ban- \\ dung, Ganesha 10, Bandung, 40132, Indonesia
}

\begin{abstract}
We analyze the spectral evolution of ultraluminous X-ray source (ULX) M82 X-1 by means of spectral fitting. We use selected Swift/XRT data in 2014 and 2015. The flux of M82 X-1 increased by a factor of 2-3 from 2014 to 2015. Most of the data in 2015 show greater dominance of hard component than those of 2014. Due to moderate signal-to-noise ratio, we only fit each spectrum with power-law and disk blackbody model separately. The data in 2014 are better fitted with powerlaw model based on the value of reduced-chi squared. On the other hand, both powerlaw and diskbb models showed comparable reduced chi-squared value for the data in 2015 . We found that the range of spectral index for 2014 data is $1.65<\Gamma<2.08$ and for 2015 data is $1.02<\Gamma<1.95$ from the powerlaw model, resembling the range for that of black hole binary system at low mass accretion rate. We obtained higher innermost disk temperature from the disk blackbody model, $1.20 \mathrm{keV}<T_{\text {in }}<3.63 \mathrm{keV}$, compared to that of black hole binary system in the thermal state. The calculated innermost radius of the disk, $R_{\mathrm{in}}$, varies between 0.99 to $4.89 R_{\mathrm{S}}$ assuming $10 M_{\odot}$ black hole which indicates that the spectral state is not in thermal dominant state but rather we suspect that M82 X-1 exhibits greater mass accretion rate than that of the thermal dominant state.

Keywords: M82 X-1, black hole accretion, spectral evolution.
\end{abstract}

\section{Introduction}

Ultraluminous X-ray sources (ULXs) are defined as point-like X-ray sources located outside the center of galaxies with luminosities exceeding $3 \times 10^{39} \mathrm{erg} \mathrm{s}^{-1}$ [3]. These apparent luminosities, assuming isotropic emission, are above the Eddington limit of a $20 M_{\odot}$ black hole. Despite the fact of being very luminous, ULXs cannot be explained by the collections of many sources with each luminosity less than the Eddington limit because many ULXs exhibit significant time variabilities [6]. One reasonable assumption is that the ULXs are single compact objects powered by accretion. If the Eddington limit applies to these objects, then ULXs is one of the best candidates for an intermediate-mass black hole (IMBH). Alternatively, ULXs may represent a class of super-Eddington emitters and probes to a regime in

\footnotetext{
*e-mail: izatulhafizah@students.itb.ac.id

**e-mail: kiki@as.itb.ac.id
} 
which the accretion rate is much higher than that seen in Galactic black hole binaries (e.g. Brightman et al. 2020)[2]. The reality is probably more complex, with some ULXs being possibly more massive than stellar-mass black holes $\left(\mathrm{M}_{\mathrm{ULX}}>100 M_{\odot}\right)$, but still experiencing the super-Eddington accretion rate. M82 X-1 is one of the brightest ULXs, which can reach $L_{\mathrm{X}} \sim 10^{41} \mathrm{erg} \mathrm{s}^{-1}$ at the distance of $3.4 \mathrm{Mpc}$ [4]. Its high luminosity, makes it one of the best intermediate-mass black-hole (IMBH) candidates, assuming Eddington-limited accretion. Detection of twin-peaked QPOs at 3.3 and $5.1 \mathrm{~Hz}$ lead to a mass estimate using scaling laws between the QPOs frequencies and mass of $428 \pm 105 M_{\odot}$ [8]. However, the mass estimates for M82 X-1 using different methods vary considerably and have large uncertainties. Therefore its status as an IMBH has not been firmly established. For instance, modelling of the accretion disk emission shows that M82 X-1 can be explained by a $\sim 30 M_{\odot}$ stellar remnant black hole radiating at several times its Eddington limit [7].

Here, we report our X-ray spectral analysis using Swift/XRT observations. We aim to investigate the variability of the X-ray spectra and estimate the mass of the black hole in M82 $\mathrm{X}$-1. In Section 2, we describe the X-ray data which we used in the this study, including the details of data reduction and fitting methods. In Section 3, fitting results are presented, together with their implications on the black hole mass. We conclude our work in Section 4.

\section{Data and Methods}

\subsection{Data}

We use Swift/XRT data for our study due to its frequent observations during 2014 and 2015. We chose 30 observation data which represent different intensity, consisting of 12 observations from 2014 and 18 observations from 2015. For 2014 data, only the observation data which have an exposure time $>5000$ seconds are used. Low exposure data are not used due to their low number of counts which render insufficient statistics. The observations in 2014 represent low intensity data. Reported by Brightman et al. (2016)[1], the flux of M82 X-1 is found to increase during May to July in 2015. Therefore, we have added 18 observations data during May to July 2015. Table 1 provides a description of the observational data.

\subsection{Data Reduction and Fitting Methods}

We extracted the source counts from a circular region with a radius of $\sim 49$ " and the background events were extracted from a nearby circular region of the same size (Figure 1). The hardness ratio is defined as the ratio between the count rate in $1.5-10 \mathrm{keV}$ to that of $0.3-1.5$ $\mathrm{keV}$.

Figure 2 shows the 0.3-10 keV count rate of each observation (light curve) and the corresponding hardness ratio. The hardness ratio shows that 2015 data are more dominated by the hard X-ray component than those of 2014. On the bottom panel, the light curve shows that the flux increases of about 2 to 3 times from 2014 to 2015. This shows a spectral evolution that is different from that of Galactic black hole binary, in which the softer component increases as the source brightened. The spectra were grouped with a minimum of one count per bin. The C-statistic was used for spectral fitting. The spectra were fitted in the range $0.3-10 \mathrm{keV}$ with two different model, powerlaw and disk black body (diskbb).

\section{Spectral Analysis}

Not all of the data can be fitted by single powerlaw or diskbb model. Table 2 shows percentage of well-fitted data to powerlaw or diskbb model for 2014 and 2015 data. The fitting 
Table 1. Observational data

\begin{tabular}{lll}
\hline Observation ID & Observation Date & $\begin{array}{l}\text { Exposure Time } \\
\text { (second) }\end{array}$ \\
\hline 33123024 & $2014-01-3100: 54: 59$ & 13456.233 \\
33123006 & $2014-01-2508: 41: 59$ & 12286.459 \\
33123023 & $2014-01-3100: 42: 13$ & 11194.395 \\
33124001 & $2014-01-2300: 48: 11$ & 9974.064 \\
33123025 & $2014-01-3118: 24: 57$ & 9812.241 \\
33123017 & $2014-01-3002: 09: 34$ & 9177.898 \\
33123063 & $2014-02-1800: 33: 59$ & 8607.612 \\
33123068 & $2014-02-2200: 34: 58$ & 7932.295 \\
33123018 & $2014-01-3016: 33: 04$ & 6423.361 \\
33123053 & $2014-02-1008: 37: 59$ & 5309.887 \\
33123035 & $2014-02-0410: 10: 59$ & 5293.636 \\
33123032 & $2014-02-0221: 40: 59$ & 5003.257 \\
92202007 & $2015-05-0503: 43: 59$ & 2020.883 \\
92202008 & $2015-05-1008: 21: 59$ & 1965.717 \\
92202009 & $2015-05-1506: 17: 59$ & 1840.302 \\
92202010 & $2015-05-2015: 35: 59$ & 1672.356 \\
92202011 & $2015-05-2516: 59: 59$ & 1747.573 \\
92202012 & $2015-05-3015: 02: 22$ & 1144.226 \\
92202013 & $2015-06-0402: 00: 59$ & 2194.543 \\
92202014 & $2015-06-1613: 01: 59$ & 1970.731 \\
81460001 & $2015-06-2017: 28: 58$ & 1648.556 \\
92202015 & $2015-06-2112: 32: 59$ & 2161.162 \\
92202016 & $2015-06-2606: 00: 59$ & 1854.772 \\
92202017 & $2015-07-0107: 02: 58$ & 1852.886 \\
92202018 & $2015-07-0617: 51: 58$ & 1766.21 \\
92202019 & $2015-07-1106: 25: 57$ & 2055.964 \\
81460002 & $2015-07-1123: 58: 58$ & 1875.44 \\
92202020 & $2015-07-1620: 35: 57$ & 2013.352 \\
92202021 & $2015-07-2104: 02: 58$ & 2124.125 \\
92202022 & $2015-07-2610: 11: 58$ & 2052.384 \\
\hline & & \\
\hline & & \\
& & \\
& &
\end{tabular}

Table 2. Well-fitted data percentage

\begin{tabular}{lll}
\hline Model & 2014 & 2015 \\
\hline powerlaw & $66.6 \%$ & $83.3 \%$ \\
diskbb & $25 \%$ & $77.7 \%$ \\
\hline
\end{tabular}

result is plotted in Figure 3. Powerlaw fittings show the range of spectral index, 1.02 to 2.08, which is similar to the range of spectral index for that of black hole binary system at low mass accretion rate. The range of $T_{\text {in }}$ values obtained from diskbb model is 1.20 to 3.63 $\mathrm{keV}$, which is higher than typical innermost temperatures of thermal state in non-rotating $\mathrm{BHB}$, but $35 \%$ of the well-fitted data gives a comparable value compared with temperatures of maximally rotating BHB.

We calculated the X-ray luminosity of M82 X-1 using the flux from fitting and assuming the distance of M82 X-1 is $3.4 \mathrm{Mpc}$. Using the innermost temperature from fitting result and luminosity that has been calculated before, we calculated the innermost radius of the disk, 


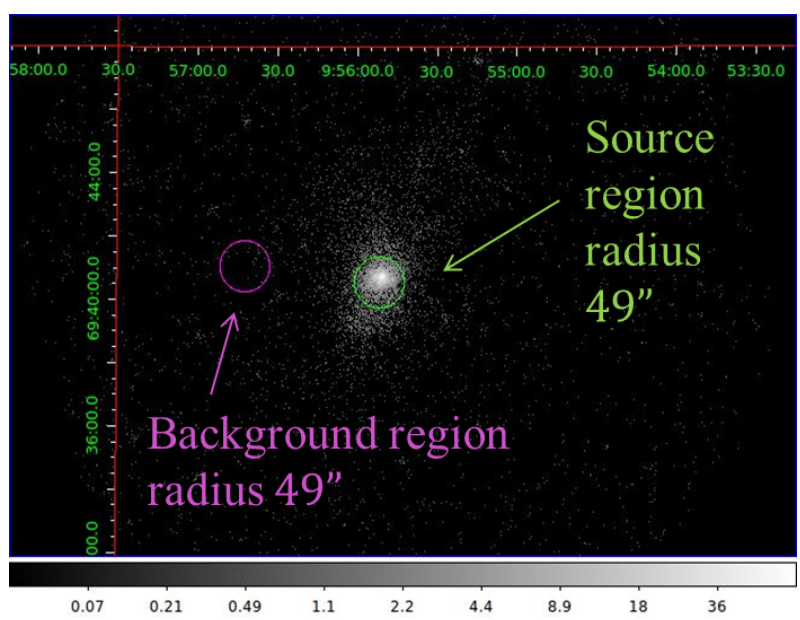

Figure 1. Swift/XRT 0.3-10 keV image of M82 X-1. The green circle shows the source extraction region and the magenta circle for background region, both have a radius of 49 ".

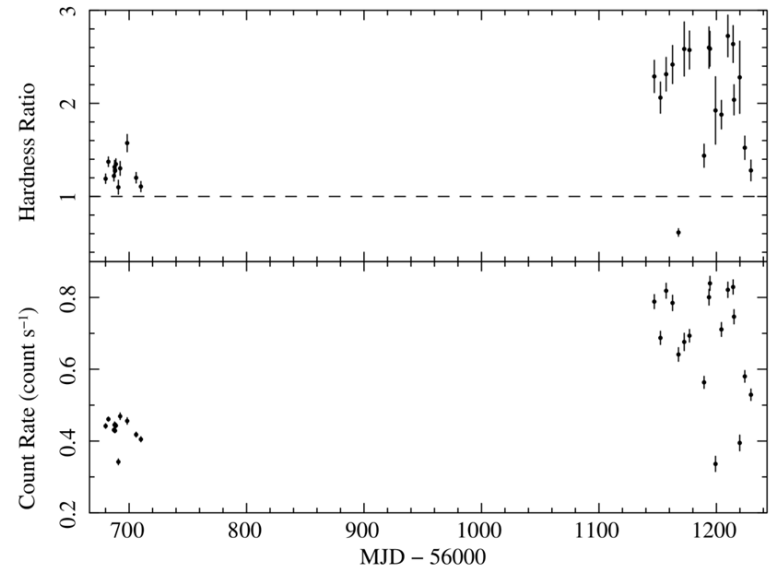

Figure 2. hardness ratio (top panel) and light curve (bottom panel).

$R_{\text {in }}$, assuming the radiation is dominated by black body radiation. We used $\kappa \sim 1.7$ [5] which is the spectral hardening factor, and $\xi=0.412$ to correct $R_{\text {in }}$. Then the mass is calculated using $M / M_{\odot}=R_{\text {in }} / 8.86 \beta$ with a $\beta$ value 1 for non-rotating and $1 / 6$ for maximally rotating black hole.

Figure 4 is temperature-luminosity plot of 2014 observations (black) and 2015 observations (red). Both 2014 and 2015 data do not show a $L \propto T^{4}$ relation which is commonly found in the thermal state of black hole binary (BHB). The data show a $L \propto T^{0.67}$ relation for 2014 and $L \propto T^{1.07}$ relation for 2015. It means the data used in this study do not follow the trend of the thermal state of BHB. This is confirmed by the radius-flux plot (Figure 5) which gives a non-constant innermost radius values. Spectrum in the thermal state will produce a more or less constant radius values. Figure 6 shows plot of radius-mass, in which a non-rotating black 

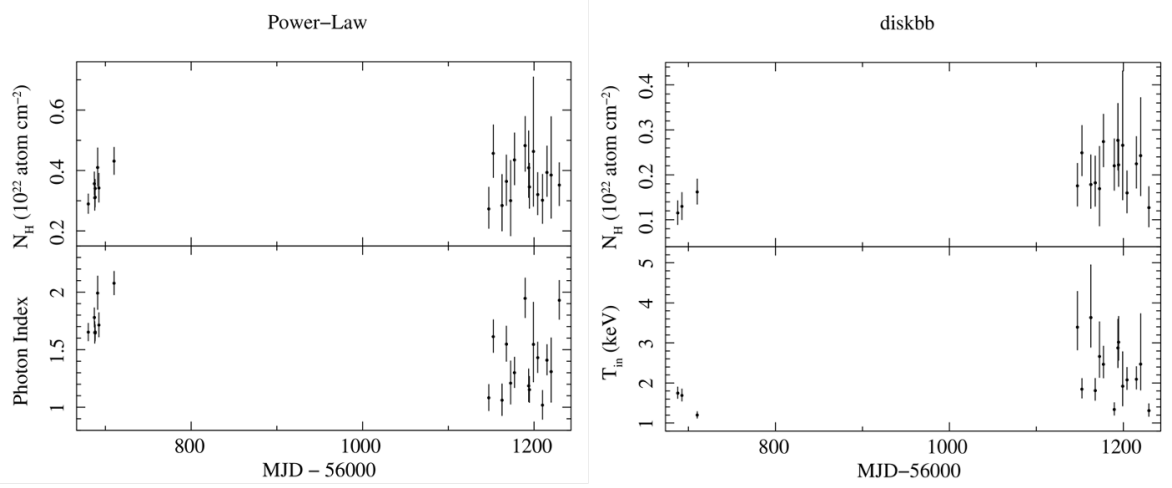

Figure 3. hardness ratio (top panel) and light curve (bottom panel).

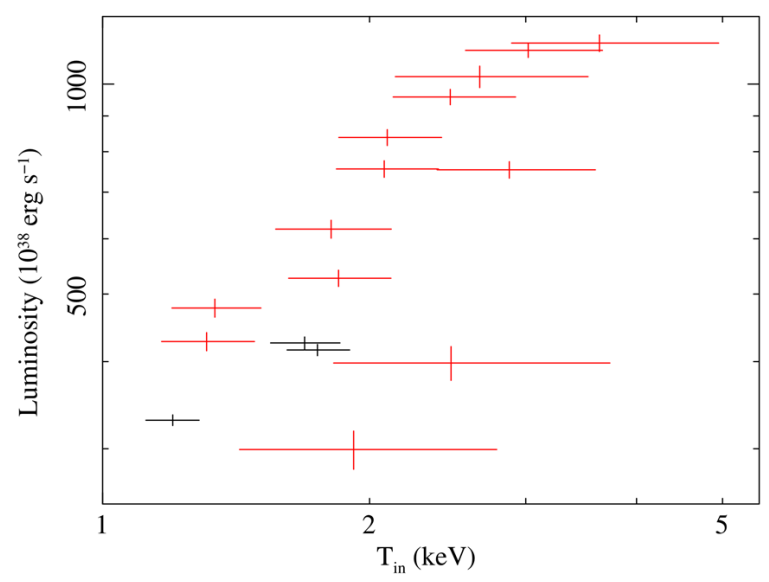

Figure 4. Temperature-luminosity plot, black for 2014 data and red for 2015 data.

hole is assumed in the data points without star symbol while maximally rotating black hole is assumed for data with star symbol. This plot shows that the obtained black hole masses varied due to the non-constant values of innermost disk radius, which cannot be physical, but instead reveal the limitation of mass estimate based on spectral fitting when the standard disk assumption cannot be justified.

\section{Conclusion}

We have used Swift/XRT data to show that the spectral variability of M82 X-1 does not resemble that of BHB. The increasing domination of hard component of the spectra as the intensity increase does not necessarily mean that the non-thermal component predominates the spectra, but may imply a higher inner disk temperature which support supercritical accretion, assuming a constant spin of the black hole. The choice of spectrum used to determine black hole mass, must be done carefully. Despite this limitation, all data used in this study indicate a black hole mass less than 100 solar mass. Based on the obtained range of black hole mass, we conclude that M82 X-1 requires a higher accretion rate to explain the different spectrum 


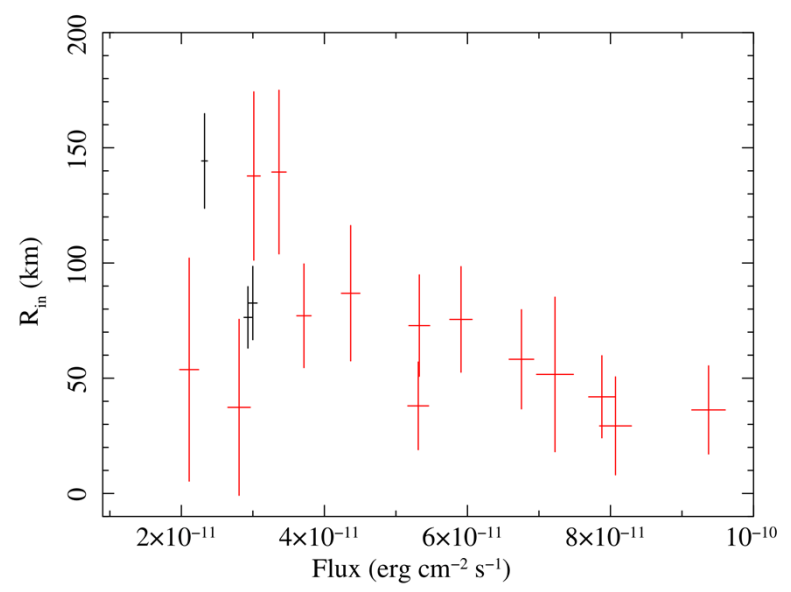

Figure 5. Flux-innermost radius plot, black for 2014 data and red for 2015 data.

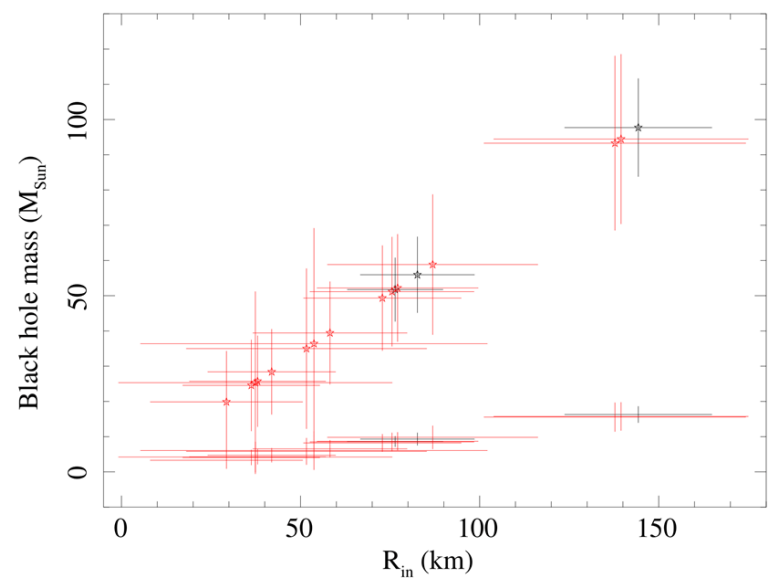

Figure 6. Radius-black hole mass plot, black for 2014 data and red for 2015 data. Star symbol for maximally rotating black hole.

transitions compared to $\mathrm{BHB}$ at sub-Eddington accretion rates. We therefore conclude that M82 X-1 is a super-Eddington accretor.

\section{References}

[1] Brightman, M., Harrison, F. A., Barret, D., et al., ApJ 829, 28 (2016)

[2] Brightman, M., Walton, D. J., Xu, Y., et al., ApJ 889, 71 (2020)

[3] Feng, H. \& Soria, R., NewAR 55, 166 (2011)

[4] Foley, R. J., Fox, O. D., McCully, C., et al., MNRAS 443, 2887 (2014)

[5] Kato, S., Fukue, J., \& Mineshige, S., Black-Hole Accretion Disks (Kyoto University Press, Kyoto, 2008) 60

[6] Makishima, K., Kubota, A., Mizuno, T., et al., ApJ 535, 632 (2000) 
[7] Okajima, T., Ebisawa, K., \& Kawaguchi, T., ApJL 652, L105 (2006)

[8] Pasham, D. R., Strohmayer, T. E., \& Mushotzky, R. F., Nature 513, 74 (2014)

[9] Poutanen, J., Lipunova, G., Fabrika, S., et al., MNRAS 377, 1187 (2007) 